\title{
RISCO DE VULNERABILIDADE DOS IDOSOS dE UMA UNIDADE dE SAÚdE dA FAMILIA NO OESTE PARANAENSE
}

\author{
Risk for old-age vulnerability of the elderly in a \\ family health unit in western Paraná
}

Giovana Balbinot ${ }^{1}$, Kurt Juliano Sack Orejuela Uscocovich².

1. Centro Universitário da Fundação Assis Gurgacz, Cascavel - PR. ORCID: https://orcid.org/00000001-7339-8839.

2. Centro Universitário da Fundação Assis Gurgacz, Cascavel - PR. ORCID: https://orcid.org/00000002-6119-6700.

CONTATO: Giovana Balbinot | Rua Presidente Bernardes, 1915 - Centro | Cascavel | Paraná | CEP 85801180 | Telefone (45) 98414-0401 | E-mail: giobalbinot@hotmail.com

COMO CITAR: Balbinot G, Uscocovich KJSO. Risco de vulnerabilidade dos idosos de uma unidade de saúde da familia no oeste paranaense. R. Saúde Públ. Paraná. 2019 Dez;2(2):13-19.

\footnotetext{
(c) (i)

COPYRIGHT Esta obra é disponibilizada nos termos da Licença Creative Commons - 4. 0

Internacional. É permitida a reprodução parcial ou total desta obra, desde que citada a fonte
}

RESUMO O presente estudo buscou avaliar o risco de vulnerabilidade dos idosos pertencentes a uma Unidade de Saúde da Família em um município do Oeste do Paraná através do uso do questionário Vulnerable Elders Survey (VES-13) estratificando estes pacientes conforme o Protocolo de Identificação do Idoso Vulnerável, através do uso do questionário VES-13. A pesquisa foi realizada de forma retrospectiva, de objetivo exploratório e descritivo de abordagem quantitativa. Foram analisados 403 idosos e observou-se que a maioria deles foram classificados como idosos não vulneráveis, predominante da faixa etária dos 60 a 69 anos e a maioria era do sexo feminino. 0 estudo conclui que os valores encontrados são satisfatórios, visto que apenas um terço dos idosos foram considerados 
vulneráveis. Ainda, foram muito similares aos dados referentes à média do estado do Paraná.

PALAVRAS-CHAVE: Assistência Integral à Saúde. Saúde do Idoso. Saúde da família. Vulnerabilidade em Saúde.

\begin{abstract}
The present study sought to evaluate the risk of vulnerability of the elderly being seen in a family health unit in a municipality in the west of the state of Paraná, using the Vulnerable Elders Survey questionnaire (VES-13) and stratifying these patients according to the Identification Protocol of the Vulnerable Elderly and the use of VES-13. The research was carried out retrospectively, with exploratory and descriptive objective and quantitative approach. A total of 403 elderly individuals were analyzed, and it was observed that most of them were classified as non-vulnerable elderly, with the age group from 60 to 69 years being predominant, and most of them being female. The study concludes that the figures found are satisfactory, since only one third of the elderly were considered vulnerable. Furthermore, out data was very similar to the average data in the state of Paraná.
\end{abstract}

KEYWORDS: Comprehensive Health Care. Health of the Elderly. Family Health. Health Vulnerability.

\title{
INTRODUÇÃO
}

0. maior acontecimento atual para a sociedade é o processo de envelhecimento que ocorre em todo o mundo'. No Brasil, esse seguimento teve início após a queda da fecundidade e mortalidade, a partir de $1960^{2}$. O número de idosos no nosso país cresce a cada aferição, estimando-se o sexto lugar no contingente de idosos no mundo, tendo 32 milhões de pessoas, em $2025^{3}$. No estado do Paraná, essa faixa etária já ocupa 11,2\% da população geral, no Censo de 2010, e a estimativa é de que até 2030 esse número chegue a 20,8\%4.

O acelerado envelhecimento dos brasileiros juntamente com o aumento da longevidade dos idosos resultou em ampliação do número de doenças crônicas e incapacidades funcionais, acarretando aumento dos custos diretos e indiretos para o nosso sistema de saúde. Isso torna uma questão atual na sociedade. Dessa forma, deve- se acompanhar a evolução do idoso, identificando potenciais pacientes de risco e no surgimento de qualquer sinal de alerta que indique fragilidade, o idoso deve ser submetido a uma investigação mais detalhada e multidimensional na Atenção Primária à Saúde ${ }^{2,5.6}$.

Essa perda da capacidade de regular a própria vida, chamada capacidade funcional, está intimamente relacionada com a queda das funções de cognição, humor e comportamento, mobilidade e comunicação. Esses processos não fazem parte do envelhecimento normal e sua manutenção é uma das causas que melhoram a qualidade de vida dessa população ${ }^{12}$. Assim, para reconhecer quais idosos estão em risco, foi preciso estratificar esse grupo para a visualização do possível problema, visar uma tomada de decisão e realizar as intervenções assistenciais e preventivas, destinando os recursos 
humanos e financeiros nos locais futuramente mais necessitados?.

O Vulnerable Elders Survey (VES-13) foi criado nos Estados Unidos e incluso na Caderneta de Saúde da Pessoa Idosa em 2014, pelo Ministério da Saúde. No estado do Paraná, desde a incorporação do questionário, em 2014, até 2017, 270 municípios já haviam aplicado o questionário e estratificado os resultados? ${ }^{7}$. Ele foi desenvolvido com o intuito de rastrear idosos vulneráveis na comunidade, isto é, pacientes com maior ameaça de redução funcional e morte ${ }^{8}$.

O escore baseia-se em dados referentes à idade do paciente, autopercepção da própria saúde, presença de limitações físicas e incapacidades. Totaliza 13 itens, sendo que cada um recebe uma pontuação específica e o somatório final varia de 0 a 10 pontos. Quanto maior o somatório de pontos, maior o risco de declínio funcional e óbito desse idoso ${ }^{2.5}$

A estratificação de risco e grau de fragilidade do idoso é feita conforme os escores obtidos com a aplicação do questionário, sendo classificado em três possíveis categorias de risco: idosos robustos, em risco de fragilização e frágeis. Os idosos robustos são aqueles que obtiveram $\leq 2$ pontos no questionário. Os idosos em risco de fragilização são os que pontuaram entre 3 e 6 e ainda conseguem gerenciar sua vida de forma autônoma, porém estão na transição do estado de senescência para a senilidade. Por último, o idoso classificado como frágil teve $\geq 7$ pontos no VES-132.

Para cada aumento de um ponto no VES13. a chance de declínio funcional ou morte nesse paciente aumenta 1,37. Os idosos vulneráveis, aqueles que pontuaram $\geq 3$, tem 4,2 vezes mais o risco de morrer ou apresentar declínio funcional ao longo de 2 anos em comparação aos idosos não vulneráveis. Em vista disso, o questionário VES-13 é eficaz para triagem e finalidade de detecção de risco de deterioração da saúde da população mais idosa e vulnerável9, 10 .

Devido ao contexto atual, é importante a estratificação de acordo com a fragilidade e a identificação dos grupos mais vulneráveis, principalmente dos idosos, a fim de modificar e adaptar os serviços e políticas públicas, aprimorar o atendimento de profissionais da saúde, gerenciar recursos financeiros e humanos, priorizando o atendimento preventivo, retardando os agravos e oportunizando melhor qualidade de vida a essa população ${ }^{8}$. Ainda, visando os idosos de baixo risco, criar estratégias de intervenções preventivas ${ }^{6}$.

Neste sentido, devido à importância do melhor cuidado da saúde dos idosos, este estudo tem como objetivo estratificar o risco de vulnerabilidade dos idosos em uma população assistida por uma USF, através da análise dos resultados da aplicação do Protocolo de Identificação do Idoso Vulnerável (VES-13) e sugerir ações de melhorias no cuidado destes.

\section{METODOLOGIA}

O presente estudo foi baseado metodologicamente em uma pesquisa retrospectiva, de objetivo exploratório e descritivo de abordagem quantitativa, por meio da avaliação através dos dados do questionário VES-13 já aplicados, entre os anos de 2015 a outubro de 2018, pela equipe de saúde aos idosos pertencentes à USF avaliada

Dos 403 idosos aos quais a equipe de saúde da família aplicou o questionário VES-13 entre os anos de 2015 a outubro de 2018, inicialmente foi dividido conforme o sexo e em três faixas etárias: 60 a 69 anos, 70 a 79 anos e 80 anos ou mais. Em seguida, agrupamos entre as classificações de risco e grau de fragilidade para termos uma visão geral e, por fim, analisamos cada grupo de forma separada para maiores detalhes.

O Projeto de Pesquisa foi submetido ao Comitê de Ética em Pesquisa com Seres Humanos do Centro Universitário FAG e aprovado dia 13 de dezembro de 2018 pelo CAAE nº 0274281 aprovado dia 13 de dezembro de 2018 pelo CAAE 
70 a 79 anos e, por fim, o grupo de 80 anos ou mais, totalizando $53 \%$, 32\% e $15 \%$, respectivamente.

Quando divididos conforme a classificação

\section{RESULTADOS}

Em uma observação geral, foram entrevistados 157 homens e 246 mulheres, constatando que o sexo feminino foi predominante com $61 \%$ de todos os entrevistados. Ainda, a maioria dos idosos fazia parte da faixa etária dos 60 a 69 anos, seguida dos de risco e o grau de fragilidade, a maior parte dos pacientes se concentrou na categoria de risco baixo/grau robusto, correspondendo a $67 \%$. seguido do risco médio/grau em risco de fragilidade com $21 \%$ e, por fim, $12 \%$ pertenceram ao risco alto/ grau frágil. Os resultados podem ser observados no Gráfico 1.

Quando analisamos isoladamente o grupo

Gráfico 1 Classificação conforme o grau de fragilidade e sexo.

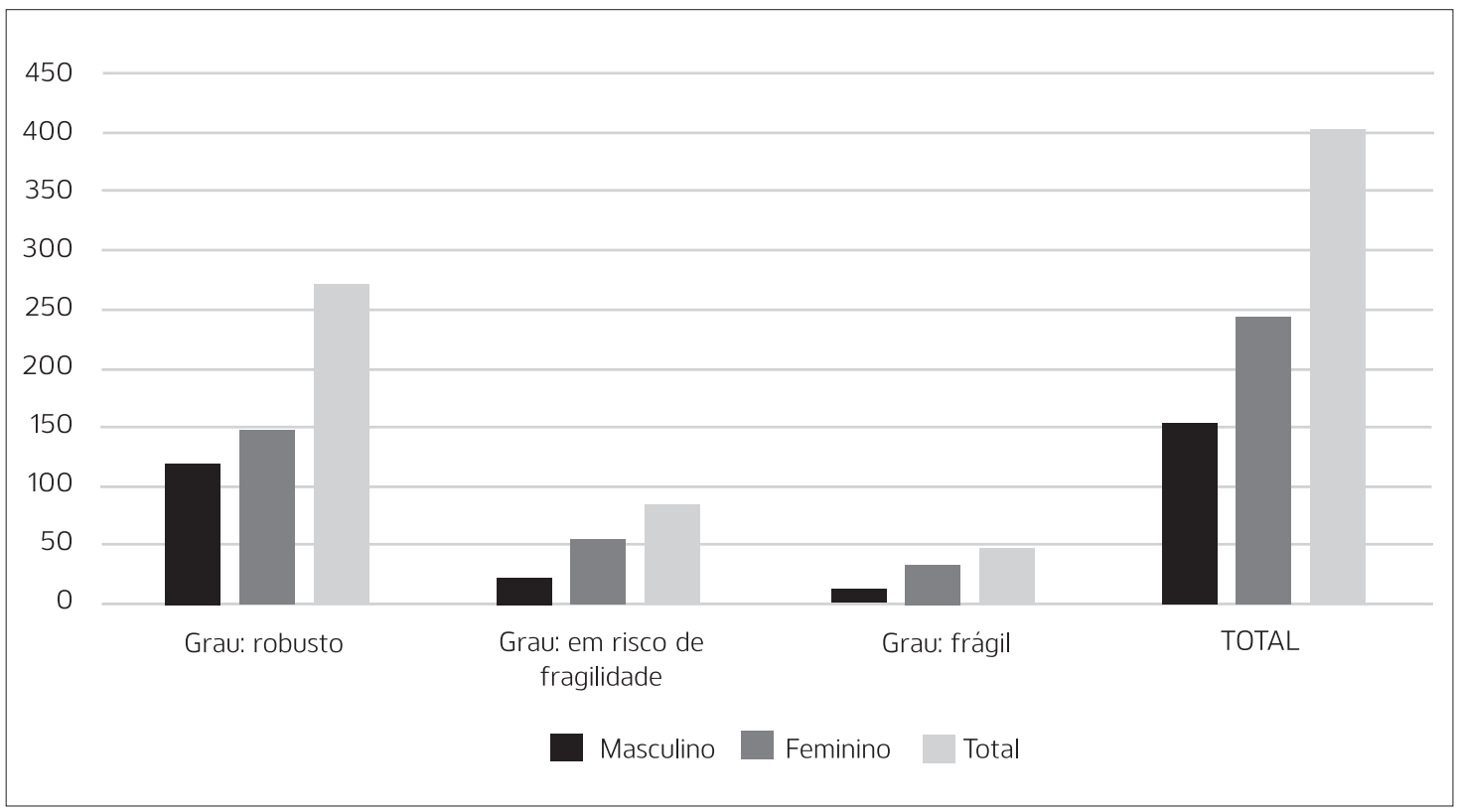

Fonte: Elaborado pelos autores (2019)

de risco baixo/grau robusto, composto por 271 entrevistados, percebemos que a porcentagem entre homens e mulheres é a mais próxima dentre os grupos estudados, representando $45 \%$ e $55 \%$, respectivamente. Pacientes de ambos os sexos são a maioria na faixa etária de 60 a 69 anos, correspondendo a $62 \%$. No intervalo de idades de 70 a 79 anos, os dois sexos revelaram a mesma porcentagem de $15 \%$ e dos 80 anos ou mais, apenas $8 \%$ dos idosos de risco baixo integram esse grupo.

Os idosos em risco de fragilização, risco moderado, foi o segundo grupo a ser explorado, mostrando predominância das mulheres em todos os intervalos de idade e arrematando $70 \%$ dos 84 pacientes examinado. A proporção de homens permaneceu a mesma entre os dois primeiros 
intervalos de idade, com $12 \%$ cada. Entre todos os segmentos analisados, o dos idosos com 80 anos ou mais classificados com risco médio obteve o menor número de pessoas, totalizando apenas 14 pacientes.

Por fim, a categoria de idosos de risco alto/ grau frágil apresenta uma diferença relevante com relação aos outros grupos: o segmento de entrevistados com 80 anos ou mais obteve a maior porcentagem dentre todos os intervalos de idade, com 54\%; seguido de 31\% entre 70 e 79 anos, e por último 15\% com idade entre 60 e 69 anos. Das 48 pessoas que integram essa categoria, 37 são mulheres e apenas 11 são homens.

\section{DISCUSSÃO}

Entre os pacientes pesquisados evidenciouse o predomínio de idosos classificados como risco baixo de vulnerabilidade com $67 \%$, pertencentes à faixa etária de 60 a 69 anos (53\%) e a maioria era do sexo feminino (61\%). Essa predominância feminina é resultado da maior expectativa de vida da mulher que, em média, vive oito anos a mais que os homens ${ }^{11}$. 39\% das mulheres eram frágeis, valor esse inferior ao estudo de Lima (2017) ${ }^{1}$ onde $56 \%$ das mulheres apresentaram algum tipo de vulnerabilidade.

Analisando o grupo predominante de risco baixo de vulnerabilidade em relação ao número total de entrevistados, 42\% tinham entre 60 e 69 anos, 20\% entre 70 e 79 anos e apenas 5\% acima de 80 anos. As mulheres predominavam no grupo, representando 55\%, porém esse nível de estratificação foi o mais próximo entre o número de mulheres ( $n=150)$ e homens ( $n=121)$.

Os pacientes em risco de fragilidade e frágil representavam $21 \%$ e $12 \%$, respectivamente, de toda a população, consequentemente totalizando um terço de pacientes vulneráveis, o que corrobora com as pesquisas ${ }^{1.12}$, onde a maior taxa foi de idosos robustos, sinalizando que a maioria dos entrevistados administravam sua vida de forma independente e sem declínio funcional.

O grupo etário no qual se pôde observar maior número de idosos que já são considerados de grau frágil foi dos entrevistados acima de 80 anos, representando 6\% do total de idosos, seguido do intervalo 70 a 79 anos com $4 \%$ e $2 \%$ eram pacientes entre 60 e 69 anos considerados como frágeis e com alto risco de vulnerabilidade. Notase que com o aumento da idade houve uma maior quantidade de pacientes frágeis.

Em um estudo sobre vulnerabilidade e envelhecimento dos idosos do município de São Paulo ${ }^{13}$, foram considerados serem de vulnerabilidade física aqueles pacientes que fizeram três pontos ou mais no questionário SABE que representava o VES-13. O estudo constatou que houve um significativo aumento do número de pessoas idosas fisicamente vulneráveis entre uma primeira análise no ano 2000 e uma segunda em 2006 (38,1\% × 52,7\%). Notou-se que a atuação do tempo poderia ser uma das explicações para essa descoberta, já que à medida que envelhecemos há uma redução da reserva funcional. Ainda, no estudo do município de São Paulo³, em 2006 83,3\% dos idosos com 80 anos ou mais apresentaram vulnerabilidade física, número esse superior aos encontrados neste estudo (65,5\%).

A pesquisa desenvolvida obteve valores muito próximos do rastreio de risco para fragilidade de idosos paranaenses realizados até março de 2017, com 264.892 idosos de 270 municípios. Estratificouse o grau de vulnerabilidade dos pacientes conforme o VES-13, obtendo os seguintes valores: $66 \%$ dos idosos eram robustos, $20 \%$ estavam em risco de fragilização e $14 \%$ eram considerados frágeis $^{14}$. Na 15a Regional de Saúde do Paraná, o rastreio teve resultados parecidos, com $71 \%$ dos idosos robustos, $16 \%$ em risco de fragilidade e 13\% frágeis $^{12}$.

Dessa forma, podemos notar que o resultado desta pesquisa, realizada com os idosos de uma USF do oeste paranaense, é condizente com a 
média dos 270 municípios do estado. Os resultados da 15a Regional de Saúde foram um pouco superior aos demais na análise da taxa de idosos robustos, com diferença de $4 \%$ para este estudo e $5 \%$ para a média estadual, porém este estudo obteve a menor porcentagem de pacientes de grau frágil, sendo 1\% a menos comparado com a 15a Regional de Saúde e $2 \%$ a menos que a média estadual.

Reconhecer os idosos vulneráveis é imprescindivel para apoiar os gestores no planejamento e evolução dos serviços e políticas de saúde ofertada à população, priorizando os recursos para o atendimento visando a promoção e prevenção, além da reabilitação absoluta e definitiva, retardando agravos, portanto, reduzindo custos $^{8,15}$. É necessário supervisionar os problemas de saúde dos idosos, a fim de estabilizar sua doença e conservar sua capacidade funcional por mais tempo possível, permitindo um envelhecimento ativo desses pacientes ${ }^{16}$.

O manejo recomendado pelo Ministério da Saúde para a Atenção Primária de Saúde (APS) é de que os idosos robustos devem manter sua independência e autonomia, fazer o manejo clínico adequando das suas comorbidades, além de promoção de saúde e prevenção primária e secundária². Deve haver busca ativa dos usuários idosos do território, aplicar o questionário VES13 e estratificar o risco dele, fazer o diagnóstico precoce de complicações e/ou condições crônicas associadas, acompanhar esse paciente na APS com consultas anuais de rotina, avaliação do cartão de vacina, educação em saúde, prevenção de doenças com foco na manutenção da capacidade funcional e promoção de hábitos saudáveis ${ }^{17}$.

Para os idosos em risco de fragilização e frágil é necessário impedir o declínio funcional desse paciente, focando na abordagem adequada da polifarmácia e polipatologia, e receber intervenções a fim de que recupere sua autonomia e independência novamente ${ }^{2}$. Devem ser feitas todas as competências descritas para os idosos com baixo risco, além de compor e assistir a realização do plano de cuidado traçado e encaminhar a outros níveis de atenção especializados quando necessário ${ }^{17}$.

\section{CONSIDERAÇÕES FINAIS}

Conclui-se que entre os pacientes pesquisados, foi possível notar o predomínio de idosos robustos, sendo a maioria da faixa etária mais jovem e do sexo feminino. Os valores encontrados em cada estratificação, conforme o Protocolo de Identificação do Idoso Vulnerável do questionário VES-13, foram muito semelhantes aos valores médios do estado. Estes resultados são satisfatórios, visto que apenas um terço dos idosos analisados são considerados vulneráveis.

Com o crescimento cada vez mais acentuado de idosos, é necessário atentar para as modificações causadas na sociedade. Deve-se acompanhar a evolução do envelhecimento, identificando sinais de perda da autonomia e transformação dos pacientes vulneráveis. Reconhecer antecipadamente os possíveis problemas identificados nessa fase auxilia para que sejam elaboradas estratégias para prevenir ou amenizar danos, bem como que sejam priorizados atenção e recursos visando manter a qualidade de vida desta população.

\section{REFERÊNCIAS}

1. Lima CAB, Carvalho JL. Aquino RCA. Avaliação de vulnerabilidade do idoso através da adaptação transcultural do instrumento de identificação do idoso vulnerável VES-13. Rev Eletrônica Estácio Recife [Internet]. 2017 Jul [citado 2019 Out 15];3(1). Disponivel em: https://reer.emnuvens.com.br/reer/article/view/115/42.

2. Paraná, Secretaria do Estado da Saúde. Oficinas do APSUS: formação e qualificação profissional em atenção primária à saúde: oficina 9: saúde do idoso na atenção primária à saúde. Curitiba: Secretaria de Estado da Saúde do Paraná; 2014 [acesso em 2019 Abr 06]. Disponivel em: http://www.saude.pr.gov.br/arquivos/File/ Apsus_cadero9_1208_14_alta.pdf. 
3. Instituto Brasileiro de Geografia e Estatística. Censo Demográfico 2010 [Internet]. Brasília: IBGE: 2010 [acesso em 2019 Abr 06]. Disponivel em: https://cidades.ibge.gov.br/brasil/pr/panorama.

4. Instituto Brasileiro de Geografia e Estatística. Projeção da população do Brasil e das unidades da federação [Internet]. Brasília: IBGE; 2019 [acesso em 2019 Abr 06]. Disponivel em: https://www. ibge.gov.br/apps/populacao/projecao//index.html.

5. Paraná, Secretaria do Estado da Saúde. Avaliação multidimensional da saúde do idoso [Internet]. Curitiba: Secretaria de Estado da Saúde do Paraná; 2018 [acesso em 2019 Abr 06]. Disponivel em: http://www.saude.pr.gov.br/arquivos/File/AvaliacaoMultiddoldoso_2018_atualiz.pdf.

6. Paz AA., Santos BRL. Eidt OR. Vulnerabilidade e envelhecimento no contexto da saúde. Acta Paul Enferm. 2006:19(3):338-42.

7. Lampert MA, Barbosa CD, Coelho FL, Santos I. Uso de preditores de risco em idosos no Brasil: uma revisão integrativa. Rev Kairos. 2016;19(3):169-85.

8. Maia FOM. Duarte YAO, Secoli SR, Santos JLF, Lebrão ML. Adaptação transcultural do Vulnerable Elders Survey -13 (VES13): contribuindo para a identificação de idosos vulneráveis. Rev Esc Enferm USP. 2012;46(esp):116-22.

9. Saliba D, Elliott M, Rubenstein LZ, Solomon DH, Young RT, Kamberg CJ, et al. The Vulnerable Elders Survey: a tool for identifying vulnerable older people in the community. J Am Geriatr Soc. 2001 Dez:49(12):1691-9

10. Min LC, Elliott MN. Wenger NS, Saliba D. Higher vulnerable elders survey scores predict death and functional decline in vulnerable older people. J Am Geriatr Soc. 2006 Mar:54(3):507-11.

11. Camarano AA. Mulher idosa: suporte familiar ou agentes de mudança?. Estud Av. 2003:17(49),35-63.

12. Paraná, Secretaria do Estado da Saúde. Estratégia de atenção à saúde do idoso: Paraná-2017 [Internet]. Maringá: Secretaria de Estado da Saúde do Paraná; 2017 [acesso em 2019 Abr 06]. Disponivel em: http://www.saude.pr.gov.br/arquivos/File/ApresentacaoEstrategiadeAtencaoaSaudedoldoso.pdf.

13. Maia FOM. Vulnerabilidade e envelhecimento: panorama dos idosos residentes no município de São Paulo - Estudo SABE [tese]. São Paulo: Universidade de São Paulo; 2011.

14. Paraná, Secretaria do Estado da Saúde. Linha guia da saúde do idoso [Internet]. Curitiba: Secretaria de Estado da Saúde do Paraná; 2018 [acesso em 2019 Abr 06]. Disponivel em: http://www. saude.pr.gov.br/arquivos/File/LinhaGuiaSaudeldoso_2018_atualiz. pdf.

15. Busato MA, Gallina LS, Téo CRPA, Ferretti F, Pozzagnol M. Autopercepção de saúde e vulnerabilidade em idosos. Rev Baiana Saúde Pública. 2015:38(3):625-35.

16. Veras RP. Prevenção de doenças em idosos: os equívocos dos atuais modelos. Cad Saude Publica. 2012:28(10):1834-40.

17. Manaus, Secretaria Municipal de Saúde. Protocolo de atenção à saúde do idoso [Internet]. Manaus: Secretaria Municipal de Saúde de Manaus; 2017 [acesso em 2018 Out 20]. Disponivel em: https://semsa.manaus.am.gov.br/wp-content/uploads/2017/09/ PROTOCOLO-IDOSO-set-2017.pdf. 